\title{
NANOSCALE FABRICATION AND MODIFICATION OF SELECTED BATTERY MATERIALS
}

\author{
Robert Kostecki, Xiang Yun Song, Kim Kinoshita, Frank McLarnon \\ Lawrence Berkeley National Laboratory \\ Berkeley, CA 94720, USA
}

\begin{abstract}
Carbon is an integral part of many battery electrodes. We explored the use of semiconductor-processing techniques that involve photolithography to pattern photoresists and subsequent pyrolysis to form carbon microstructures that function as microelectrodes. In this study, we describe the status of the fabrication of carbon microelectrodes obtained by pyrolysis of photoresist. Electrochemical nanometer-scale patterning of the surface of a conducting lithium manganese oxide $\left(\mathrm{LiMn}_{2} \mathrm{O}_{4}\right)$ by scanning probe microscopy (SPM) was studied. We show that a localized surface chemical change can be confined to a depth which depends on the oxide-tip voltage difference and ambient humidity The ability to produce nanometer-size patterns of chemically modified oxide or nanometer-sized alterations of the oxide morphology is demonstrated and discussed with reference to possible mechanisms.
\end{abstract}

\section{INTRODUCTION}

Carbon is an integral part of many battery electrodes. In some electrodes, it plays a passive role as a conductive matrix (e.g., positive electrode containing metal oxides), and in others it is an active component as an electrocatalyst (e.g., positive electrode in Zn/air cells) or as the host for electroactive species (e.g., $\mathrm{Li}^{+}$ions in negative electrode for $\mathrm{Li}$-ion batteries). Because carbons are available in a wide variety of morphologies and crystallographic structures, there are many opportunities to use carbon in battery applications. One approach that we are exploring is the use of semiconductor-processing techniques that involve photolithography to pattern photoresists and subsequent pyrolysis to form carbon microstructures that function as microelectrodes [1-4]. In this paper, we describe the status of the fabrication of carbon microelectrodes obtained by pyrolysis of photoresist and characterization of the carbons by surface-sensitive techniques and electrochemical analysis by cyclic voltammetry. Both carbon films and microelectrode structures were fabricated from pyrolyzed photoresist, and electrochemical results obtained with these carbon materials will be presented

The idea of generating patterned structures directly on a substrate has many advantages versus traditional indirect lithography techniques which involve multiple-step procedures. The ability to position the probe tip with sub-nanometer precision has stimulated efforts to use scanning probe microscopes (SPMs) to fabricate nano-scale features on surfaces [5-10]. SPMs have been used for nanometer-scale lithography on ultra-thin polymer films [11], patterning by local oxidation of hydrogen-passivated silicon surfaces [12], fabrication of single-electron transistors by the anodic oxidation of $\mathrm{Nb}$ [13], micro-contact printing [14], and dip-pen nanolithography [15]. However, a significant 
problem encountered when attempting to fabricate nano-features with conventional atomic force microscopy (AFM) or scanning tunneling microscopy (STM) is the presence of a thin water layer adsorbed or condensed onto the substrate. Such a water layer can interfere with the probe tip motion, however it exhibits very high conductivity [16] and serves as a medium for highly localized electrochemical processes [17].

\section{EXPERIMENTAL}

Both positive (AZ-4330, Hoechst Celanese, Somerville, NJ and OIR-897, Olin Corp., Norwalk, CT)) and negative (XPSU, Microchem. Corp., Newton, MA) photoresists were pyrolyzed to form carbon structures. Except for the final heat-treatment steps, the procedures involving patterning and photolithography were carried out in the Microfabrication Facility in the Electronics Research Laboratory on the campus of the University of California at Berkeley.

Raman spectroscopy measurements were carried out to investigate carbon film structure and a surface profile analyzer (NewView System 200, Zygo Corp., Middlefield, $\mathrm{CT}$ ), which combines optical microscopy and interferometry was used to obtain images of the microstructure and 3-D surface topography.

Redox couples, such as $\mathrm{I}_{3}{ }^{-/ \mathrm{I}^{-}}$and $\mathrm{Fe}(\mathrm{CN})_{6}{ }^{4-} / \mathrm{Fe}(\mathrm{CN})_{6}{ }^{3-}$, were used to investigate the electrochemical response of the carbon materials. A more detailed description of the electrochemical cell and experimental conditions is presented by Kostecki et al. [2,4].

We used an AFM to both image and modify the surface of the conducting oxide $\mathrm{LiMn}_{2} \mathrm{O}_{4}$ in air [17]. The AFM tips were coated with thin layers of Pt or $\mathrm{W}_{2} \mathrm{C}$. All AFM experiments were performed in constant-force mode with controlled oxide-tip voltage differences. A single scan of the tip over the oxide surface simultaneously produced two images: a topographic image and a conductance image; the latter represents oxide-tip current variations during scanning at a given oxide-tip voltage difference. Thin $(\sim 400 \mathrm{~nm})$ films of $\mathrm{LiMn}_{2} \mathrm{O}_{4}$ were deposited onto Pt or stainless-steel substrates [17].

X-ray diffraction patterns and Raman spectra of the $\mathrm{LiMn}_{2} \mathrm{O}_{4}$ and thin films confirmed a cubic spinel structure with a Fd3m space symmetry group and a $0.819 \mathrm{~nm}$ cubic cell lattice parameter. Raman microscopy measurements were carried out with a LabRam microscope made by ISA Groupe Horiba.

\section{RESULTS}

\section{(i) Carbon Microstructured Electrodes}

We have developed a fabrication procedure based on semiconductor processing techniques to produce carbon microstructures that were evaluated in electrochemical studies. This effort involved identifying a precursor material and the appropriate processing steps to produce the carbon microstructure. In addition, the technique to form the microstructure was developed by using photolithography and patterning. Both the interdigitated carbon electrodes and 3-D posts were fabricated by the semiconductor processing steps illustrated in Fig. 1. In the first step, a thin layer of $\mathrm{Si}_{3} \mathrm{~N}_{4}$ (about $100 \mathrm{~nm}$ ) is deposited by chemical vapor deposition (CVD), which serves as an insulator to separate 

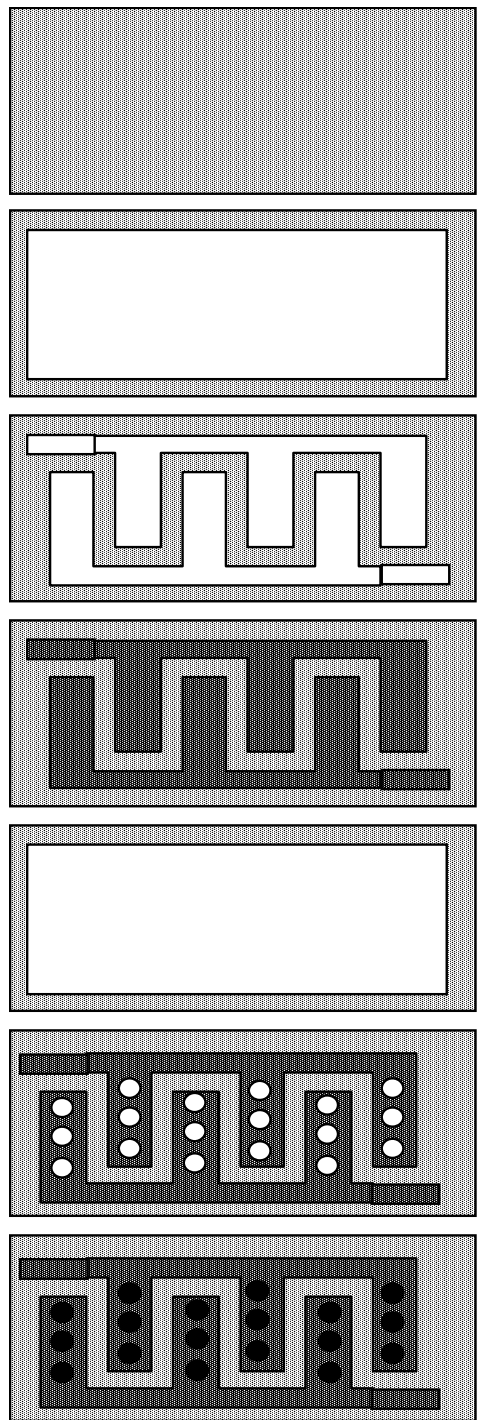

\section{CVD of Si 3N4 layer on Si wafer}

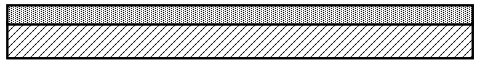

Spin coat photoresist on Si3N4/Si wafer

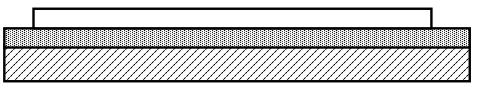

Pattern current collector by photolithography

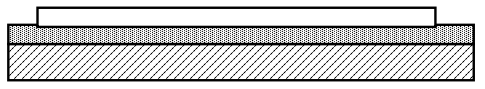

Cure and bake

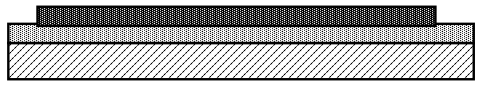

Spin coat second layer photoresist

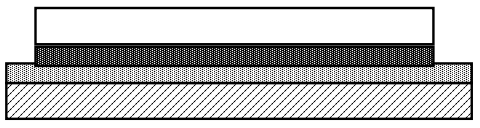

Pattern post structure by photolithography

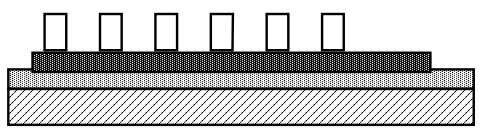

Heat treat to form carbon post structure

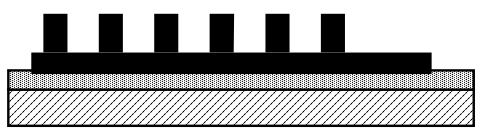

Figure 1. Processing steps to form 3-D carbon microelectrodes

the conductive silicon wafer from the carbon structure. Two sequential steps involving spin coating, patterning, curing, baking and pyrolysis of the photoresist are employed to form the final carbon microstructure. In the first sequence, the interdigitated fingers are formed, then the process is repeated, except a different mask is used to pattern the phototresist to form the posts. In the figure, white represents the photoresist before heat treatment. The carbon obtained by heat treatment is depicted by dark grey and black The step involving pyrolysis of the photoresist is critical to obtain the desired carbon microstructure. As a prelude to this step, experiments were conducted to produce carbon films from the photoresist that was spin coated on Si wafers. Usually, the patterned photoresist film was carbonized for 1 hour at temperatures between 600 to $1100^{\circ} \mathrm{C}$ to form smooth carbon microstructures. We have previously reported [3] on the preparation of carbon films, which can serve as the current collectors, and the electrochemical results obtained on these films. The carbons obtained from pyrolyzed photoresist have properties similar to those of disordered carbons. 
An example of the carbon microstructure obtained from the processing steps outlined in Fig. 1 is presented in Fig. 2. A surface profile analyzer was used that combines optical microscopy and interferometry to obtain images of the microstructure and 3-D surface topography. The image in Fig. 2 clearly shows the structure of the carbon posts arranged in a regular array on the fingers. The posts are approximately $3 \mu \mathrm{m}$ in

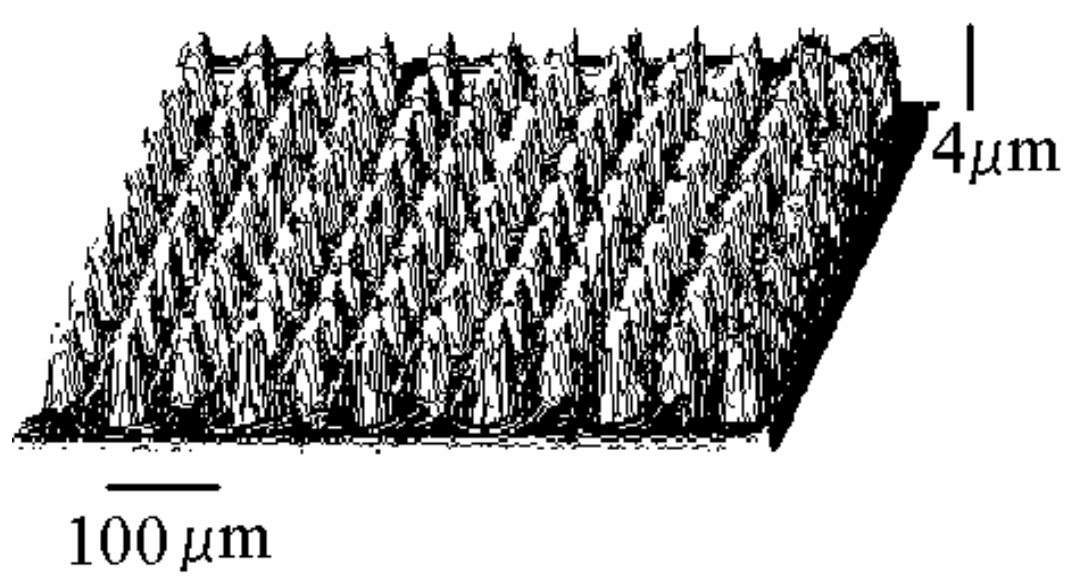
height above the carbon fingers and their width at the base is about $40 \mu \mathrm{m}$ in diameter. Although the patterned photoresist is initially cylindrical, the carbon that is formed has a more conical structure, indicative of the shrinkage and decomposition that occurs upon heat treatment

Electrochemical studies were conducted to characterize the carbon electrodes by redox reactions involving the iodine/iodide redox couples. The close arrangement of the carbon fingers yields an electrochemical response that is similar to that of a RRDE for detecting ionic species in solution. In order to evaluate quantitatively the collection coefficient of the Interdigitated Array Electrodes (IDAEs) of different size and geometry a series of amperometric measurements were carried out. A slow $10 \mathrm{nA} / \mathrm{s}$ current scan from 0 to $5.9 \mu \mathrm{A}$ was

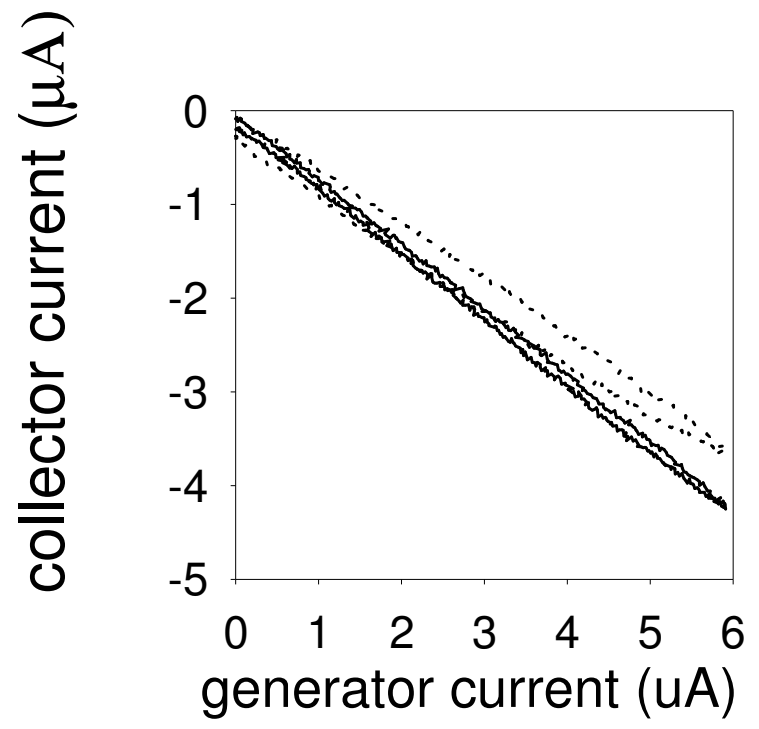
implemented on the generator electrode

Figure 3. Electrochemical response of a carbon asymetric IDEA electrode. Solid line - inner electrode as a generator, dotted line - outer electrode as a generator. Slow $10 \mathrm{nA} / \mathrm{s}$ current scan from 0 to $5.9 \mu \mathrm{A}$ was implemented on the generator. Collector electrode was held at $-0.1 \mathrm{~V}$.

to produce $\mathrm{I}_{3}{ }^{-}$ions that were reduced back to $\mathrm{I}^{-}$at the collector electrode which was polarized potentiostatically at $-0.1 \mathrm{~V}$. The results obtained for the iodine system with the asymetric microelectrode geometry are shown at Figure 3. The increase in the anodic current at the generator electrode during the scan produces a cathodic current response of a similar magnitude at the collector electrode. The cathodic current at the collection electrode shows a slight hysteresis with a higher current observed during the reverse potential scan on the generator electrode. The contribution from $\mathrm{I}_{3}{ }^{-}$ions that escaped and accumulated in the electrolyte during the anodic 
scan is responsible for this behavior. The generator-collector relationship shows very good linearity within 0-5 $\mu \mathrm{A}$ current range, and the collection efficiency can be easily determined from the slope of the current line. We found that the collection efficiency of the carbon IDAEs varied from c.a., 60 to $90 \%$ depending on size and geometric arrangement of carbon electrodes.

The initial results presented here illustrate the electrochemical response of the pyrolytic carbon IDAEs to redox reactions, and demonstrated IDAEs collection efficiencies are comparable to multiband IDAEs. This study demonstrates that semiconducor-processing techniques are a promising and viable approach to producing carbon microelectrode for miniaturized electrochemical systems.

\section{(ii) Electrochemical nanolitography of manganese oxides}

Figure 4A shows a standard topographic AFM image of a representative $5 \times 5 \mu \mathrm{m}$ area of a $\mathrm{LiMn}_{2} \mathrm{O}_{4}$ surface. A conductance image, represented by the oxide-tip current response of the same area (not shown here) at a $-1.0 \mathrm{~V}$ (i.e., oxide is poised negative vs. the AFM tip) voltage difference between the oxide and AFM tip, displayed a uniform current distribution. The current was fairly stable and did not exceed $1.5 \mathrm{nA}$. Its magnitude was determined by the electronic properties of the oxide and the tip, the voltage difference, and the specific geometry of both the tip and the oxide surface.

However, experiments with the AFM and $\mathrm{LiMn}_{2} \mathrm{O}_{4}$ at ambient conditions and at different oxide-tip voltage differences led us to an observation that the current tended to decrease upon repeated scans over a given area, and it decreased rapidly with time when the tip was immobilized. Importantly, the rate of current decrease at a positive voltage differences was rapid, the current was steady and slow at negative oxide-tip voltage difference.

We then selected five separate areas within the same $5 \times 5 \mu \mathrm{m}$ region, and we scanned each of them once at $+1.0 \mathrm{~V}$. Figure $4 \mathrm{~B}$ shows a subsequent AFM image, recorded at $-1.0 \mathrm{~V}$, of the same $5 \times 5 \mu \mathrm{m}$ region. The conductance was substantially reduced in the areas scanned at a positive voltage difference with no visible change in the oxide surface morphology. In contrast to imaging at a negative voltage, a positive voltage caused a rapid decline of film
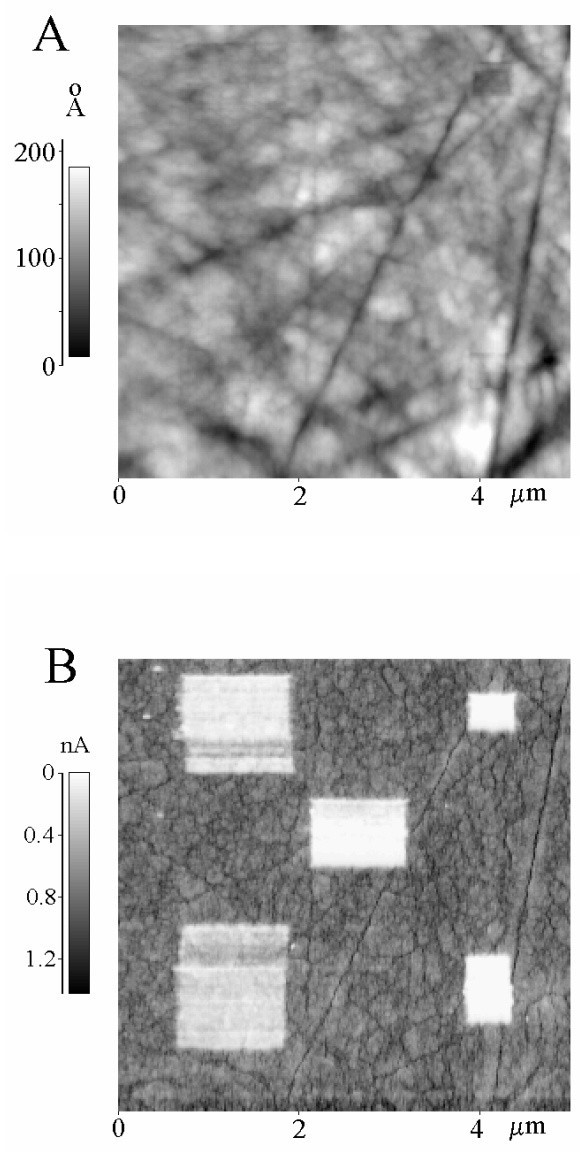

Fig.4. AFM images of surface topography (A) and conductance (B) of a $\mathrm{LiMn}_{2} \mathrm{O}_{4}$ surface (5 X 5 $\mu \mathrm{m})$ at ambient conditions, recorded at $-1.0 \mathrm{~V}$ after surface modification of five areas produced at $+1.0 \mathrm{~V}$. 
conductance. The fact that the surface conductance declines irreversibly at both positive and negative voltage differences at different rates suggests a similar mechanism, albeit with different process efficiencies. However, by reducing the magnitude of the negative voltage difference to $<0.1 \mathrm{~V}$ we could suppress the resistance buildup while preserving good contrast of the conductance image. These findings demonstrate that we can use a positive oxide-tip voltage difference to modify the local conductance of the oxide in a controlled manner, i.e., we can "write" with a negatively charged tip, and then "read" the as-produced pattern with a positively charged tip. We made several successful attempts to modify areas $\sim 20 \mathrm{~nm}$ wide, however the relatively high surface roughness made it extremely difficult to generate modified areas of regular geometric shape.

Regarding the mechanisms by which AFM modifies the $\mathrm{LiMn}_{2} \mathrm{O}_{4}$ surface electronic properties, the observed asymmetric oxide-to-tip voltage dependence renders abrasion, local heating, and hot-electron-induced reactions rather unlikely but lends strong support to electrochemical processes. The existence of a thin layer of adsorbed water on most materials under ambient conditions has been demonstrated by STM experiments on both conductive and non-conductive samples [16].

To investigate the role of adsorbed water, a series of experiments in a $\mathrm{N}_{2}$ atmosphere at controlled relative humidity $(\mathrm{RH})$ levels was carried out. The effect of humidity on the rate of surface modification was dramatic and in direct proportion to the water vapor content. At RH levels close to saturation, the oxide-tip current did not vanish after a scan at positive voltage but remained high during several tens of scans over the same area. Figure 5 presents a set of $5 \times 5 \mu \mathrm{m}$ AFM images (A - topography, B - conductance) recorded at $-1.0 \mathrm{~V}$ following 40 scans at $+1.0 \mathrm{~V}$ of a $2 \times 2 \mu \mathrm{m}$ area located in the middle of the $5 \times$ $5 \mu \mathrm{m}$ region. This experiment was carried out in $\mathrm{H}_{2} \mathrm{O}$-saturated $\mathrm{N}_{2}$ atmosphere. The topographic image shows that scanning at $+1.0 \mathrm{~V}$ at high $\mathrm{RH}$ led to localized etching of the $\mathrm{LiMn}_{2} \mathrm{O}_{4}$ surface.

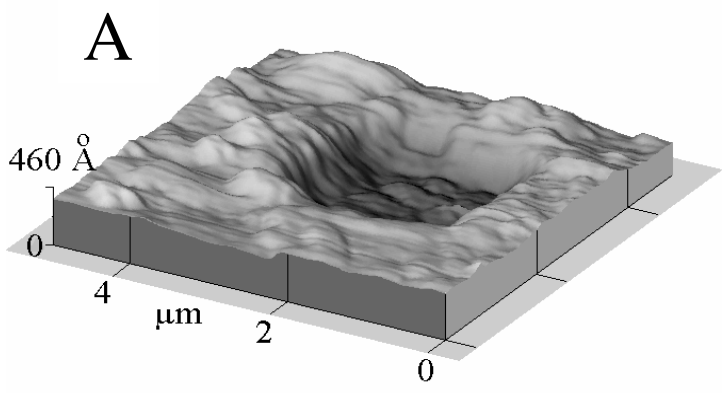

B

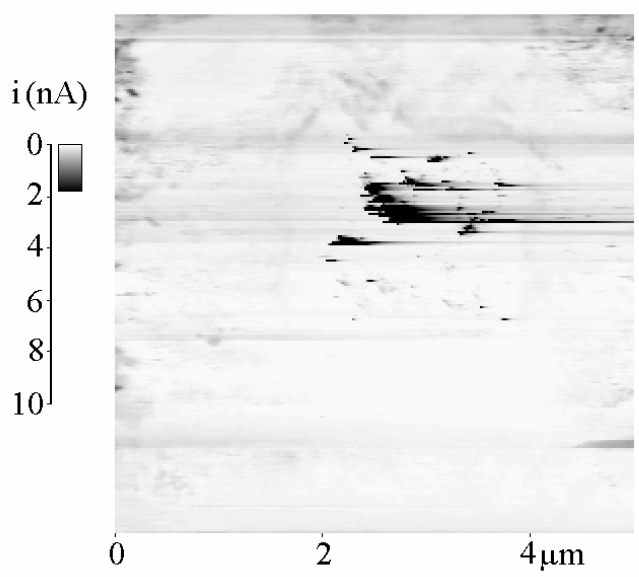

Figure 5. AFM images of surface topography (A) and conductance (B) of a $\mathrm{LiMn}_{2} \mathrm{O}_{4}$ surface recorded at $-1.0 \mathrm{~V}$. Surface-localized etching was induced by AFM scanning within a $2 \times 2 \mu \mathrm{m}$ region (center area of the $5 \times 5 \mu \mathrm{m}$ image) at $+1.0 \mathrm{~V}$ in a $\mathrm{H}_{2} \mathrm{O}$-saturated $\mathrm{N}_{2}$ atmosphere after 40 scans. Prolonged scanning at positive voltage etched a cavity $50 \mathrm{~nm}$ deep. The morphology of the region which encircles the $2 \times 2 \mu \mathrm{m}$ area was also modified, mainly due to accumulation of reaction products which were removed from the cavity. The conductance image shows a dramatic increase of the surface resistance not only in the $2 \times 2 \mu \mathrm{m}$ area but also in the surrounding region. The area of very low conductance spread over the entire AFM viewing 
window, suggesting that the reaction products formed in the hole were dragged away from the cavity by the AFM tip and spilled over the entire region.

The non-conductive character of the reaction products not only renders them detectable by electron-probe methods such as conducting-probe AFM and STM but also limits the reaction penetration depth into the oxide at low-to-moderate ambient RH. The $\mathrm{LiMn}_{2} \mathrm{O}_{4}$ film undergoes a shallow surface transformation at low $\mathrm{RH}$ with little or no detectable morphology change. When the water content at the oxide surface is high, e.g., at high $\mathrm{RH}$, the reaction products (which may be either water-soluble or hygroscopic) can be penetrated by the tip and dragged away from the scanned area, thereby exposing fresh $\mathrm{LiMn}_{2} \mathrm{O}_{4}$ material. The observed mounds on the sides of the cavity we created suggest that an electrochemical process combined with abrasive etching created the cavity.

Current-voltage $(i$-V) curves of an immobilized AFM tip in contact with the oxide were reported in [17]. We found that in the absence of water layer (zero relative humidity environment) the current is basically limited only by the resistance of the film. The substantial current increase in the presence of adsorbed water is consistent with a contribution from electrochemical reactions, especially at high voltage. The non-conductive reaction products rapidly formed an electronic barrier between the stationary tip and the oxide.

The writing-reading process and the $i$-V curves exhibited very limited sensitivity to the tip material. In a series of AFM experiments with a $\mathrm{W}_{2} \mathrm{C}$-coated tip very similar and reproducible results were obtained, however the surface modification process proceeded at a slightly higher positive voltage. An interfacial resistance or a small gap between the tip and the sample (Fig. 6) must cause the oxide-to-tip voltage difference to increase until an electrochemical process could arise at overpotentials specific for the tip and oxide. The resulting electrochemical reactions will be limited to an area determined by the size of the water meniscus between the tip and oxide, as well as the electric field and current distribution in the close vicinity of the tip.

\section{An in situ Raman microscopy}

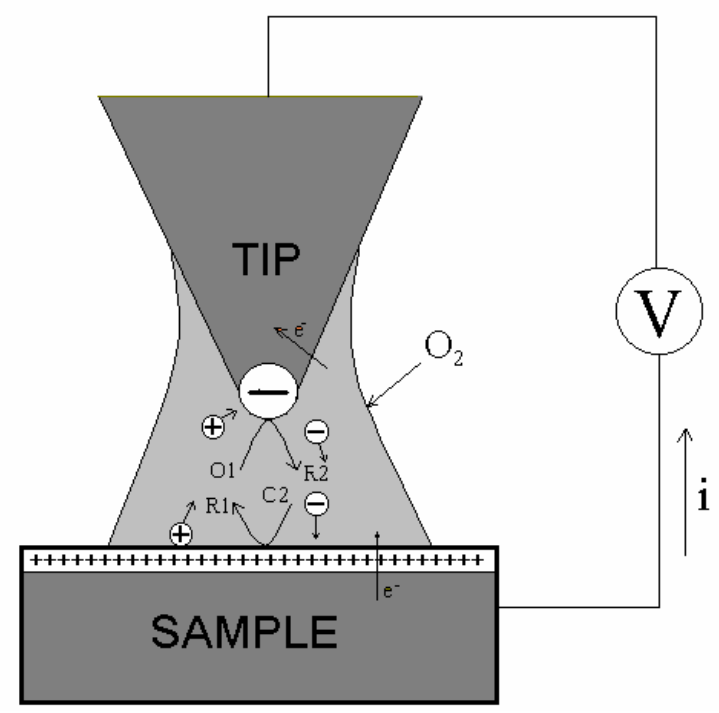

Figure 6. Schematic representation of localized electrochemical processes which occur between the sample and a conductive AFM tip experiment was carried out to identify local electrochemical reaction products. The AFM tip was replaced with a thin $0.1 \mathrm{~mm}$ dia. Pt wire and its sharp end was brought in contact with the $\mathrm{LiMn}_{2} \mathrm{O}_{4}$ electrode. The ambient relative humidity was nearly $100 \%$ in this case. A potential difference of $2 \mathrm{~V}$ between the $\mathrm{LiMn}_{2} \mathrm{O}_{4}$ sample and the $\mathrm{Pt}$ wire was applied, and the electrode surface at the point of contact was observed through the optical microscope and the current response was monitored. The current diminished very quickly from the initial value and vanished completely after 40-50 s of polarization. When the current dropped to zero the Pt wire was removed and Raman microscopy analysis of the modified region was carried out. 
Figure 7 shows an optical microscope image of the reaction region which was created in the $\mathrm{LiMn}_{2} \mathrm{O}_{4}$ thin film by the Pt wire at $2 \mathrm{~V}$. The dark area in the image center represents a cavity which was formed at the point of where the Pt wire contacted the sample.

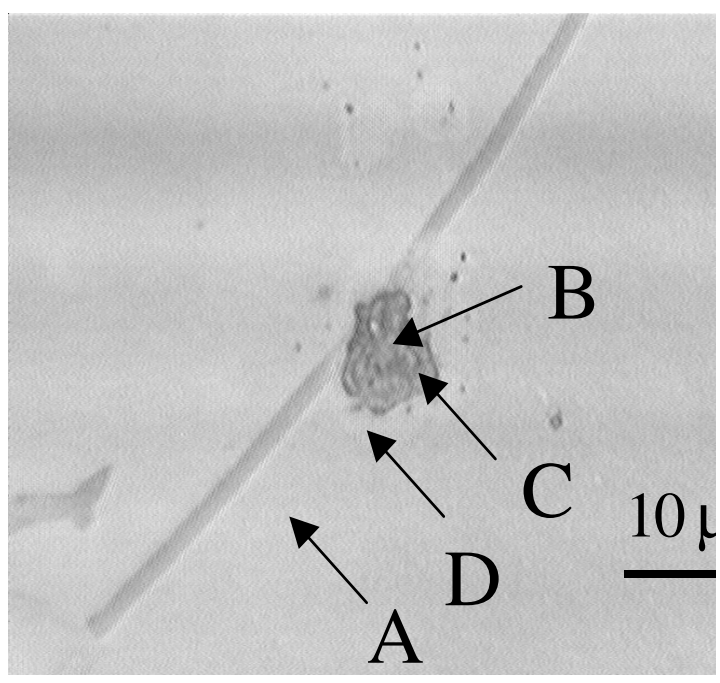

Figure 7. An image of the reaction zone between the Pt tip and the $\mathrm{LiMn}_{2} \mathrm{O}_{4}$ sample. The dark cavity in the image center represents the point of contact. Arrows mark surface sites at which Raman spectra were recorded.

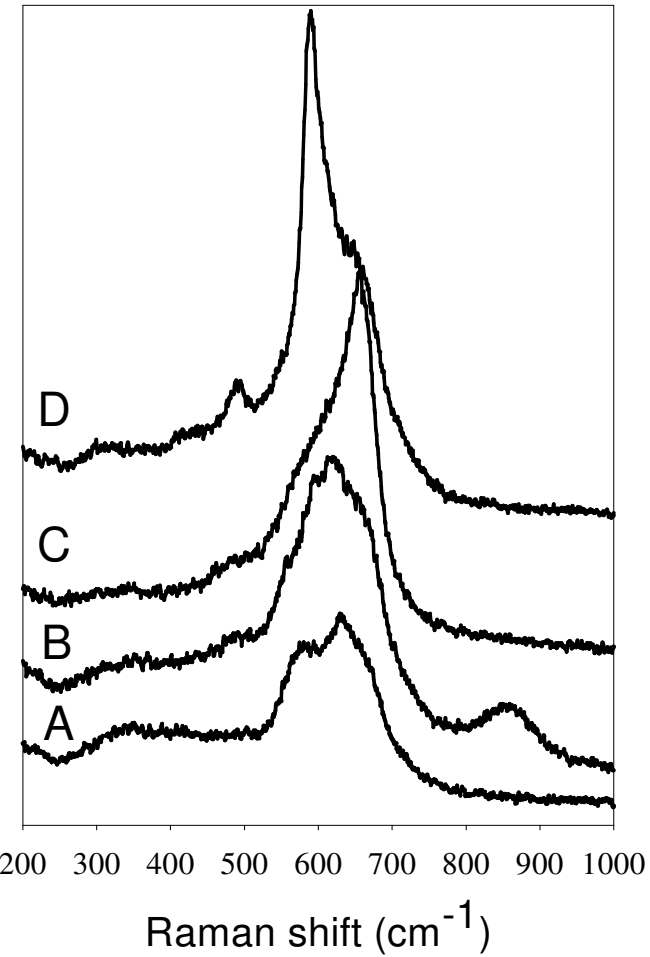

Figure 8. Raman spectra recorded of the reaction zone. Original material (A), bottom of the cavity (B), inner edge of the cavity (C), outer edge of the cavity (D).

Four Raman spectra were recorded from the reaction zone and its vicinity (Fig. 8). The spectrum of the original material (A) is typical for the $\mathrm{LiMn}_{2} \mathrm{O}_{4}$ spinel and consists of a broad band centered at $630 \mathrm{~cm}^{-1}$ and two shoulders at 590 and $655 \mathrm{~cm}^{-1}$. The Raman spectrum taken from the bottom of the cavity (B) shows very similar features however, slight band broadening is observed. Also, a new weak and broad band located at $860 \mathrm{~cm}^{-1}$ becomes visible. Dramatic changes in the lithium manganese oxide composition are observed as the Raman probe approaches the edge of the cavity. The spectrum measured at the inner part of the edge (C) shows a strong band at $655 \mathrm{~cm}^{-1}$, which is characteristic for $\mathrm{Mn}^{3+}$ oxides and hydroxides. Surprisingly, the spectrum recorded at the outer edge of the cavity (D) in the bright shadow zone which surrounds the cavity displays a sharp peak at $590 \mathrm{~cm}^{-1}$ which is characteristic for $\lambda-\mathrm{MnO}_{2}$ [18].

It is still difficult to identify the specific electrochemical reactions responsible for modification of the original film at positive (anodic) voltage. Given the products of the $\mathrm{LiMn}_{2} \mathrm{O}_{4}$ conversion, candidate reactions include the direct oxidation of $\mathrm{LiMn}_{2} \mathrm{O}_{4}$ to $\lambda-\mathrm{MnO}_{2}$ and $\mathrm{Li}_{2} \mathrm{O}$ or $\mathrm{LiOH}$ and the simultaneous reduction of water and/or protons at the tip. However, the formation of only $\lambda-\mathrm{MnO}_{2}$, which preserves the original cubic spinel structure of $\mathrm{LiMn}_{2} \mathrm{O}_{4}$, cannot explain the observed film dissolution and production of at 
least partially soluble manganese compounds at high RH. Another conceivable mechanism involves water oxidation at the $\mathrm{LiMn}_{2} \mathrm{O}_{4}$ film, which can lead to a local decrease of $\mathrm{pH}$ and subsequent disproportionation of $\mathrm{LiMn}_{2} \mathrm{O}_{4}$ into $\mathrm{Mn}(\mathrm{II})$ and $\lambda-\mathrm{MnO}_{2}$ [18]. Thus, the original $\mathrm{LiMn}_{2} \mathrm{O}_{4}$ structure will break down, and $\mathrm{Mn}$ (II) and $\mathrm{Li}$ ions will contribute to the solution meniscus ion conductivity and/or precipitate in the form of salts and/or hydroxides to form a non-conductive electronic barrier. The thickness and structure of the water layer (which depend on $\mathrm{RH}$ ) determine the extent and rate of this process.

In summary, we demonstrated the use of contact AFM for nanometer-scale electrochemical lithography of thin $\mathrm{LiMn}_{2} \mathrm{O}_{4}$ films. Although we used only $\mathrm{LiMn}_{2} \mathrm{O}_{4}$ films in this study, this novel approach may be used to develop electrochemical reaction schemes to image and modify other inorganic and organic materials which may be used for the direct fabrication and detection of nanometer-scale patterns.

\section{ACKNOWLEDGMENT}

This work was supported by the Director, Office of Energy Research, Office of Basic Energy Sciences, Chemical Sciences Division of the U.S. Department of Energy under Contract No. DE-AC03-76SF00098 and the Berkeley-France Fund.

\section{REFERENCES}

1. J. Kim, X. Song, K. Kinoshita, M. Madou and R. White, J. Electrochem. Soc., 145, 2314 (1998).

2. R. Kostecki, X. Song and K. Kinoshita, Electrochem. and Solid-State Lett., 2, 461 (1999).

3. K. Kinoshita, X. Song, J. Kim and M. Inaba, J. Power Sources, 81-82, 170 (1999).

4. R. Kostecki, X. Song and K. Kinoshita, J. Electrochem. Soc., 147, 1879 (2000)

5. G.M. Shedd and P.E. Russel, Nanotechnology, 1, 67 (1990)

6. "Electrochemical Nanotechnology", edited by W.J. Lorenz and W. Plieth, Wiley-VCH, 1998.

7. D.M. Eigler and E.K. Schweizer, Nature, 344, 524 (1990)

8. I-W. Lyo and Ph. Avouris, Science, 253, 173 (1991)

9. H.J. Mamin, P.H. Guethner and D. Rugar, Phys. Rev. Lett., 65, 2418 (1990).

10. Y. Utsugi, Nature, 347, 747 (1990)

11. M.A. McCord and R.F. Pease, J.Vac.Sci Technol. B6, 293 (1988)

12. J. A. Dagata, J. Schneir, H. H. Harary, C. J. Evans, M. T. Postek and J. Bennet, Appl. Phys. Lett., 56, 2001 (1990)

13. J-I. Shirakashi, K. Matsumoto, N. Miura and M. Konagai, Appl. Phys. Lett., 72, 1893 (1998)

14. A. Majumdar, P.I. Oden, L.A. Nagahara, J. J. Graham and J. Alexander, Appl. Phys. Lett., 61, 2293 (1992)

15. R. D. Piner, J. Zhu, F. Xu, S. Hong and C.A. Mirkin, Science, 283, 661 (1999)

16. R. Gluckenberger, M. Heim, G. Cevc, H. F. Knapp, W. Wiegräbe and A. Hillebrand, Science, 266, 1538 (1994)

17. R. Kostecki and F. McLarnon, Appl. Phys. Lett., 76, 2536 (2000)

18. J. C. Hunter, J. Solid State Chem., 39, 142 (1981) 